\title{
Gradhiva
}

GRADHIV

Revue d'anthropologie et d'histoire des arts

$14 \mid 2011$

Carl Einstein et les primitivismes

\section{La statuaire des mers du Sud, 1926}

\section{Carl Einstein}

Traducteur : Isabelle Kalinowski

\section{OpenEdition}

Journals

Édition électronique

URL : http://journals.openedition.org/gradhiva/2203

DOI : $10.4000 /$ gradhiva.2203

ISSN : 1760-849X

Éditeur

Musée du quai Branly Jacques Chirac

Édition imprimée

Date de publication : 30 novembre 2011

Pagination : 189-193

ISBN : 978-2-35744-046-3

ISSN : 0764-8928

\section{Référence électronique}

Carl Einstein, «La statuaire des mers du Sud, 1926 », Gradhiva [En ligne], 14 | 2011, mis en ligne le 30

mai 2012, consulté le 20 avril 2019. URL : http://journals.openedition.org/gradhiva/2203 ; DOI :

10.4000/gradhiva.2203

(c) musée du quai Branly 


\section{La statuaire des mers du Sud}

Le texte qui suit est la traduction d'un texte allemand rédigé par Carl Einstein en 1926 pour une exposition d'art océanien à la galerie Flechtheim de Berlin. Seul le texte introductif est présenté ici. Il était suivi d'un catalogue illustré de 26 planches photographiques; Einstein avait rédigé les notices, généralement très brèves, des 184 objets exposés, issus de la collection particulière de Flechtheim et originaires pour la plupart des anciennes colonies allemandes de Nouvelle-Guinée. Une première traduction française, illustrée, parut en juillet 1926 dans la revue L'Amour de l'art, sous le titre "Sculptures mélanésiennes", et une traduction anglaise fut publiée dans The Art en 1927. Pour un commentaire et une mise en contexte de cet essai, on se reportera à l'article de Philippe Peltier, "L'art océanien entre les deux guerres : expositions et vision occidentale», Journal de la Société des océanistes XXXV, 65, 1979, p. 271-282.

Les sculptures de la collection Flechtheim proviennent des anciennes colonies allemandes de l'archipel Bismarck - à savoir la Nouvelle-Guinée allemande, la Nouvelle-Poméranie (Nouvelle-Bretagne), le NouveauMecklembourg (Nouvelle-Irlande) et le NouveauHanovre.

Ces œuvres d'art sont nées dans le cadre d'une culture de la pierre. On fait usage de haches de pierre, de couteaux et de poignards d'os, d'obsidienne ou de coquille. On ne s'étonnera pas que, avec le choc terrible de la colonisation, l'introduction brutale de la civilisation européenne et le sabotage complet du monde spirituel et religieux ancien, cet art avant tout destiné à remplir des finalités religieuses se soit éteint.

Rien ne peut plus efficacement contredire la superstition évolutionniste que ce fait : c'est au moment où les conditions de vie se sont incontestablement améliorées et où les outils sont devenus plus performants que les vestiges de la culture en place ont dégénéré et disparu avec une effrayante rapidité.

Les indigènes de l'archipel Bismarck vivent entourés de forces magiques et de démons. Leur forme sociale est le matriarcat, c'est-à-dire que la lignée sanguine maternelle prédomine. Comme dans d'autres lieux, le droit matriarcal est associé à l'exogamie: c'est-à-dire que nul ne peut s'allier à une femme du même clan ou totem. Le totem est plus important que l'individu, et grâce à ce dernier et aux forces qui l'en- tourent, on espère que l'alliance avec un autre animal totémique (par le mariage) va apporter un surcroît de forces magiques. Nous croyons moins aux théories de l'inceste qui restent malgré tout des théories moralisantes à base biologique.

L'insigne totémique de la mère est généralement transmis à ses enfants. Comme la société, la nature, les esprits des ancêtres et les forces totémiques sont divisés sur un mode totémiste, et la différenciation et la multiplication continuelle des démons conduit à un éclatement religieux et psychique du moi auquel correspond le plus souvent une incapacité à former des communautés tribales de taille relativement importante. L'inquiétude et le tourment liés à cette trop grande différenciation sont compensés par un conservatisme rassurant, le culte des ancêtres. Le présent est divisé à l'infini. Sans cesse apparaissent de nouvelles forces magiques contre lesquelles on invente des mesures défensives; la vie de ces êtres de l'âge de la pierre, menacés de sortilèges, est écrasée entre ces antagonismes magiques, dont les conflits et les luttes de tribus sont l'expression. Des esprits des morts que l'on célèbre et que l'on se concilie au cours de longues cérémonies s'immiscent dans le présent de façon menaçante; une bonne partie des arts sculptés trouvent leur origine dans le culte des ancêtres.

$\mathrm{Au}$ matriarcat s'opposent comme presque partout des sociétés masculines dont les coutumes, les outils 


\section{TEXTES DE CARL EINSTEIN}

magiques, les lieux de cérémonie, les maisons et les cultes ne doivent pas être vus par les femmes et passent pour tabous.

Une bonne partie de ces sculptures viennent des maisons des sociétés masculines et des temples aux ancêtres. Il faut avouer que les signes de cet art symbolique sont restés jusqu'ici à peu près incompréhensibles, pour l'essentiel, ou qu'on n'a guère dépassé le stade de l'interprétation la plus vague. Tout signe ornemental a une signification précise, mais le sens est déjà devenu incertain pour les indigènes eux-mêmes, et plusieurs personnes interprètent le même ornement de façon tout à fait différente. À cela s'ajoute le fait que les œuvres des sociétés secrètes étaient évidemment cachées et que l'essentiel, à savoir le sens magique conférant le pouvoir, était anxieusement tenu secret par leurs propriétaires. Peut-être était-ce là la raison pour laquelle les œuvres d'art étaient si souvent détruites : d'abord parce qu'elles perdaient leur force magique après usage dans les cérémonies; mais aussi, peut-être, parce qu'on préférait détruire ces objets magiques que les laisser à la portée de mains indésirables.

Presque toute la sphère de la réalisation artistique est un artisanat d'hommes et, souvent, ces objets sont créés par des artistes identifiés comme tels. Peut-être regarde-t-on la statue d'ancêtre comme le siège de l'âme errante. En réalité, au demeurant, les représentations des indigènes ont rarement été claires pour nous. Par moments, on pourrait penser à la croyance dans une sorte de double de l'âme : comme si une âmeimage entrait dans la statue tandis que l'autre figure d'âme séjournait ou errait dans un autre lieu.

Le jeune indigène devient digne d'entrer dans une société secrète quand il a vécu dans la brousse, séparé de sa famille, en compagnie de garçons du même âge -identifiés peut-être à des esprits -, et été initié pour la première fois aux traditions magiques de sa communauté. Peut-être ces jeunes gens vivent-ils alors comme des sortes d'esprits des morts, ce que suggèrent leurs peintures corporelles; l'entrée dans la société secrète signifie alors une sorte de résurrection perçue, dans le cycle de la nature, comme une impulsion nouvelle et célébrée comme une consolation. L'influence de ces sociétés secrètes est immense, car elles se flattent d'être en possession de forces et d'instruments magiques.

Tournons-nous d'abord vers les figures d'ancêtres de Nouvelle-Guinée. Surmonté de l'oiseau totémique protecteur, le crâne est le plus souvent mis en valeur plastiquement, tandis que le corps est traité comme une partie entièrement plate. Dans certaines de ces figures, curieusement, le corps est incisé comme un support ornemental. Je risque ici une interprétation que l'on pourra discuter : les crânes étaient très appréciés par les défunts et conservés avec les squelettes dans des huttes. Lorsqu'on changeait de hutte, on emportait les restes des défunts. Peut-être ce support ornemental renvoyait-il au squelette des ancêtres. L'importance frappante accordée aux crânes est peut-être le vestige d'un culte des crânes ancien. Pensons aux figures d'ancêtres de la baie de Geelvink (Nouvelle-Guinée hollandaise) : le crâne du défunt est souvent placé dans leurs têtes monumentales, en sorte que la tête sculptée est le masque de l'ancêtre. Les masques sont la demeure spacieuse des esprits des ancêtres, et le bruit retentissant des bull-roarers fait entendre leur voix. Je signalerai en outre que le support-squelette des figures d'ancêtres de Nouvelle-Guinée allemande rappelle peut-être les balustrades korwar de la baie de Geelvink. La mise en valeur plastique de la tête suggère peut-être que l'ancêtre est représenté comme un porteur de masque. Peut-être faut-il interpréter les danses masquées de la même façon, peut-être signifient-elles une danse des ancêtres enfermés dans les masques.

De la même façon, nous croyons que, dans le cas des oiseaux totémiques sculptés que les danseurs doivent tenir à la main, l'important est la danse de l'oiseau totémique et non le danseur.

L'indigène de l'archipel Bismarck fait figurer sur beaucoup d'objets usuels et d'armes des sculptures qui représentent des masques et des animaux totémiques; il nous semble hors de doute que cette coutume recèle un sens magique. En Nouvelle-Guinée, les boucliers sont sculptés de masques d'épouvante, semblables aux têtes de Méduse, ou ornés de symboles qui représentent et signifient toujours quelque chose de précis et sont peut-être destinés à capter certaines forces dans les armes d'attaque et de défense.

Cette collection offre de riches exemples de deux types de masques bien distincts. Le premier est le masque à long nez que l'on rencontre fréquemment à l'embouchure du fleuve Ramu et dans l'île Zwanzigmeileninsel; le second est un type au visage large qui orne souvent les boucliers et se prête surtout à un usage décoratif et ornemental.

L'art du Nouveau-Mecklembourg, de NouvellePoméranie et du Nouveau-Hanovre se caractérise par une ornementation plus complexe et une orientation foncièrement dramatique: nous sommes surpris par l'inquiétude graphique des monumentales figures uli du Lamasong et des statues de craie du mont Rossel. 


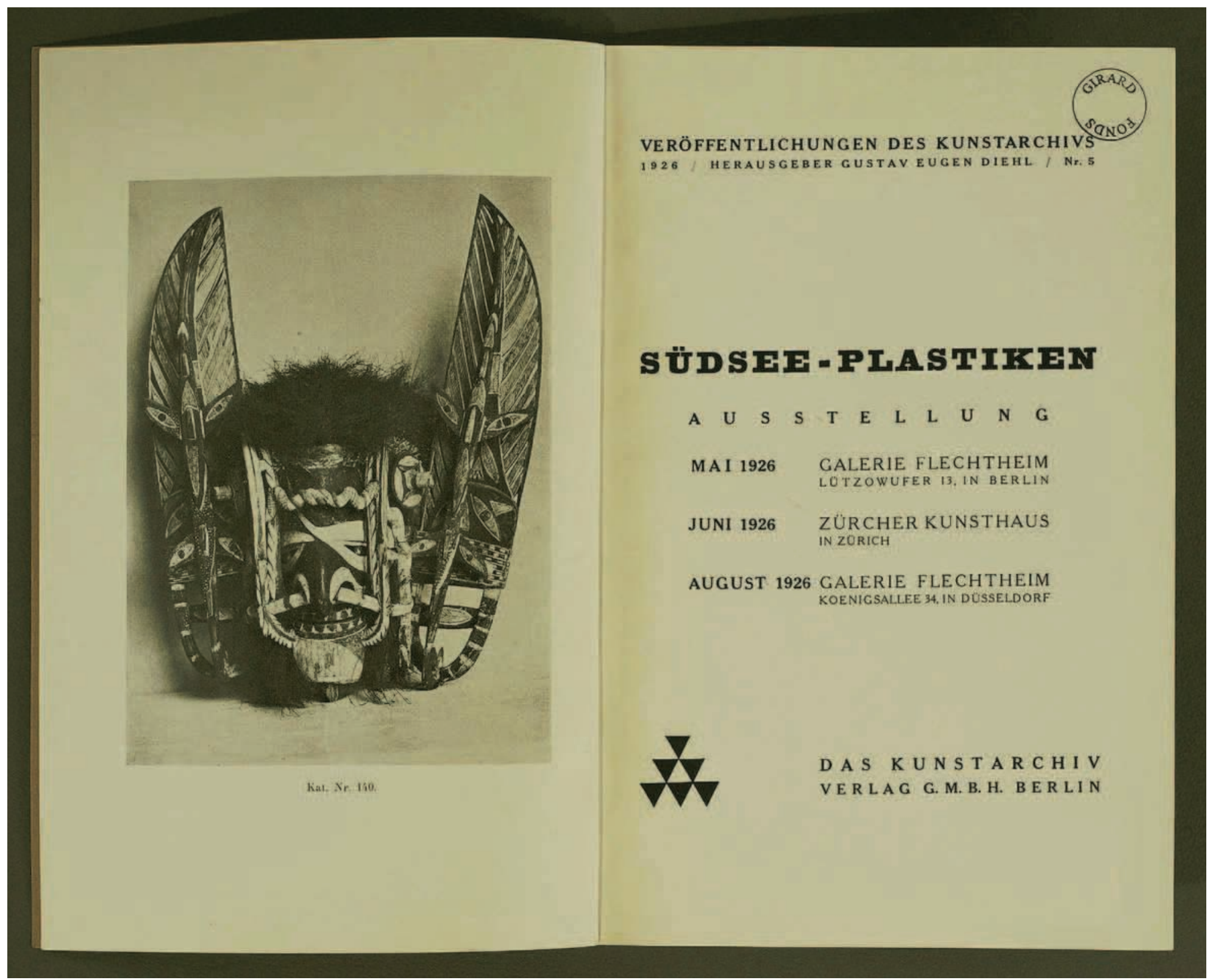

Fig. 3 Catalogue de l'exposition Südsee-Plastiken en mai 1926 à la galerie Flechtheim (Berlin), en juin 1926 à la Zürcher Kunsthaus (Zürich), en août 1926 à la galerie Flechtheim (Düsseldorf). Photo Alberto Ricci. 


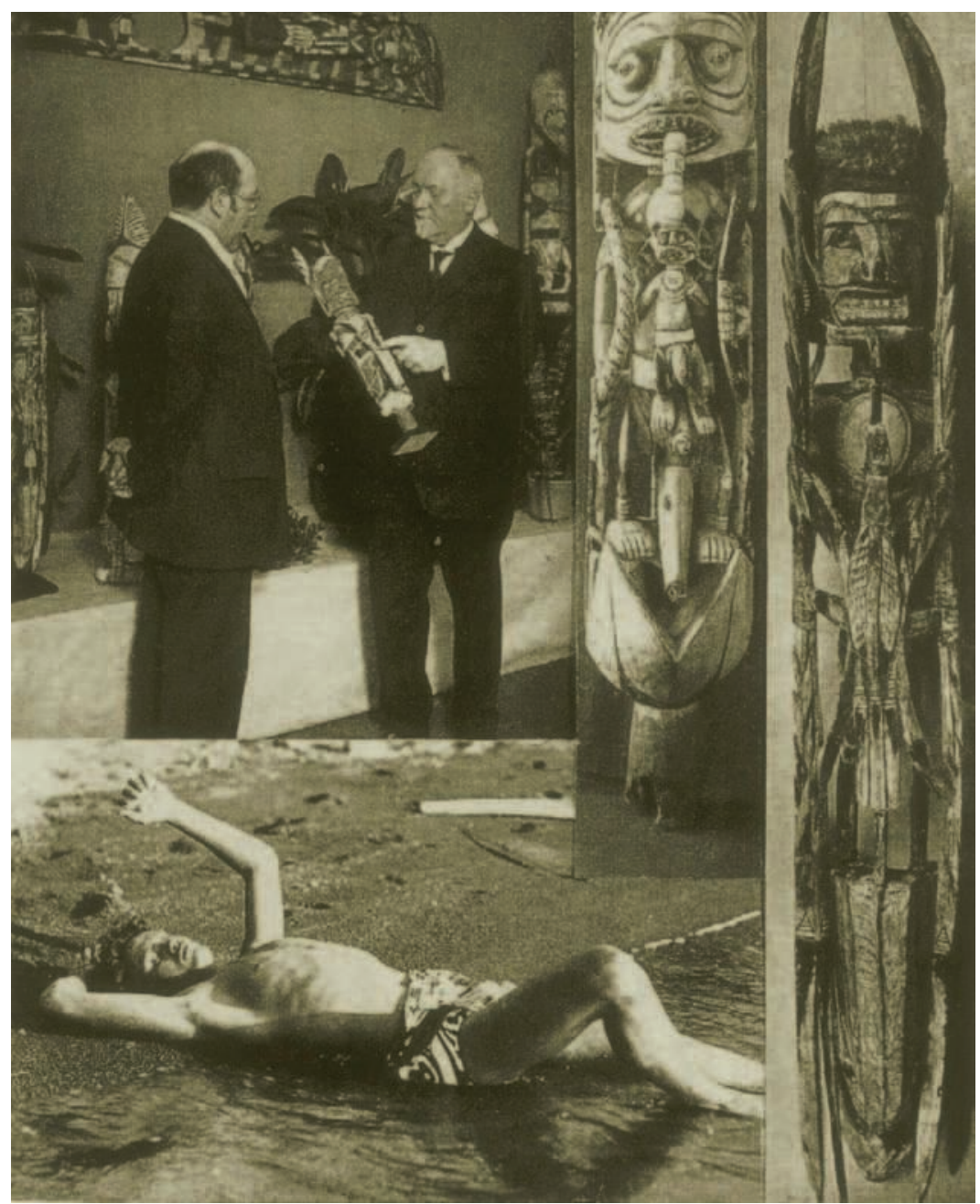

Fig. 4 Carl Einstein et August Eichhorn, directeur du Völkerkunde Museum de Berlin, regardant la collection d'art océanien d'Alfred Flechtheim, in Omnibus. Almanach auf das Jahr 1932.

Les masques de ces îles ont surtout été étudiés par Parkinson, qui s'est employé de façon méritoire à en décrire les différents types. Il distingue notamment les masques tatanua, des masques-heaumes coiffés de cheveux-chenilles renversés sur le sommet de la tête; le corps est enveloppé dans des vêtements de feuilles et les masques servent à exécuter des scènes de pantomime en l'honneur des défunts. On représente par exemple le combat des calaos ${ }^{1}$ avec les serpents, au cours duquel les danseurs du totem du rhinocéros tiennent entre leurs dents des têtes de rhinocéros sculptées.

Ces masques sont conservés dans des maisons de masques soigneusement gardées, et ils sont tabous pour les femmes et les enfants. Au-dessus des tatanua, on entrepose les masques kepong; ces masques richement sculptés ne sont pas portés pour la danse, mais comme des parures; ceux qui les revêtent tiennent un bâton dans une main, un hochet de coquillages dans l'autre, et ils s'en vont de maison en maison, sans prononcer une parole, pour quêter de la monnaie de coquillages destinée à financer les banquets de cérémonie. Les masques tatanua portent presque toujours la coiffure de deuil typique; le crâne est rasé sur les côtés et la partie médiane est ornée d'une crête hérissée colorée de jaune. Les parties rasées sont recouvertes d'un ciment dans lequel on modèle soigneusement des ornementations. De façon frappante, les masques tirent souvent la langue; sans doute le crachat remplit-il une fonction magique de protection. Non sans analogie avec le support-squelette des figures d'ancêtres de Nouvelle-Guinée, ces masques-heaumes font surtout ressortir la structure osseuse de la tête, tout en la réinterprétant librement sur un mode ornemental. Le masque kepong porte toujours le manu (l'oiseau) du défunt, son insigne totémique, qui permet de reconnaître le clan de l'ancêtre et indique en même temps sous une forme imagée le lien totémique qui unit à ce dernier et à l'animal du clan. De la même façon, on peint souvent l'animal totémique sur le corps comme un ornement propitiatoire. Les masques kepong représentent déjà l'épopée du combat du totem et des animaux totémiques. Le manu protège contre le mauvais démon des serpents ennemis qui l'encerclent. Mais l'art du Nouveau-Mecklembourg, de la Nouvelle-Poméranie et du Nouveau-Hanovre ne peut pas se contenter d'une telle représentation épique des combats des démons, 
des mythes et des fables. Le modelé est plus agité et plus varié sur les frontons matua - des bas-reliefs de grande taille que l'on salue par de bruyantes lamentations. D'autres sculptures, comme les bois sculptés kulibu et totok, ne sont jamais exposées mais conservées dans des huttes soigneusement protégées. Mentionnons encore les sculptures turu, qui restent dans une tonalité tout à fait dramatique et représentent le plus souvent des événements mythiques.

Nous pouvons observer, peut-être, une certaine affinité entre les sculptures turu et la planche cultuelle de Nouvelle-Guinée ${ }^{2}$.

À ces sculptures complexes, percées de toutes parts, s'opposent les monumentales figures uli et les sculptures de craie. Elles ne sont pas détruites après les cérémonies comme les autres malanggane, mais enveloppées et conservées dans les maisons des hommes. Augustin Krämer distingue douze sortes de figures du type uli et il a décrit les longues cérémonies en l'honneur des morts dont ces sculptures forment le cœur. Les figures uli sont à n'en pas douter des figures d'ancêtres. Certains voient dans les uli des figures bisexuelles à cause de leurs seins proéminents; Krämer, lui, interprète cette caractéristique comme un signe d'opulence et de richesse. On trouvera des détails sur les statues de craie dans le catalogue.

Il n'est guère d'art qui expose avec une telle force la tension et le déchirement démoniques de ces insulaires, qui reflète en tout point le morcellement de la société. Le culte extatique des ancêtres, les retrouvailles permanentes avec les morts et la présence effrayante de la magie et des esprits autour des vivants ont conduit cet art à représenter une forme d'agitation extrême, apaisée çà et là par le motif de la parenté entre l'homme et la nature, les animaux totémiques, amis et vénérés.

\section{$\bullet$}

1. En allemand, Nashornvogel ou « oiseau-rhinocéros 》.

2. Einstein se référait ici à des objets présentés dans l'exposition.

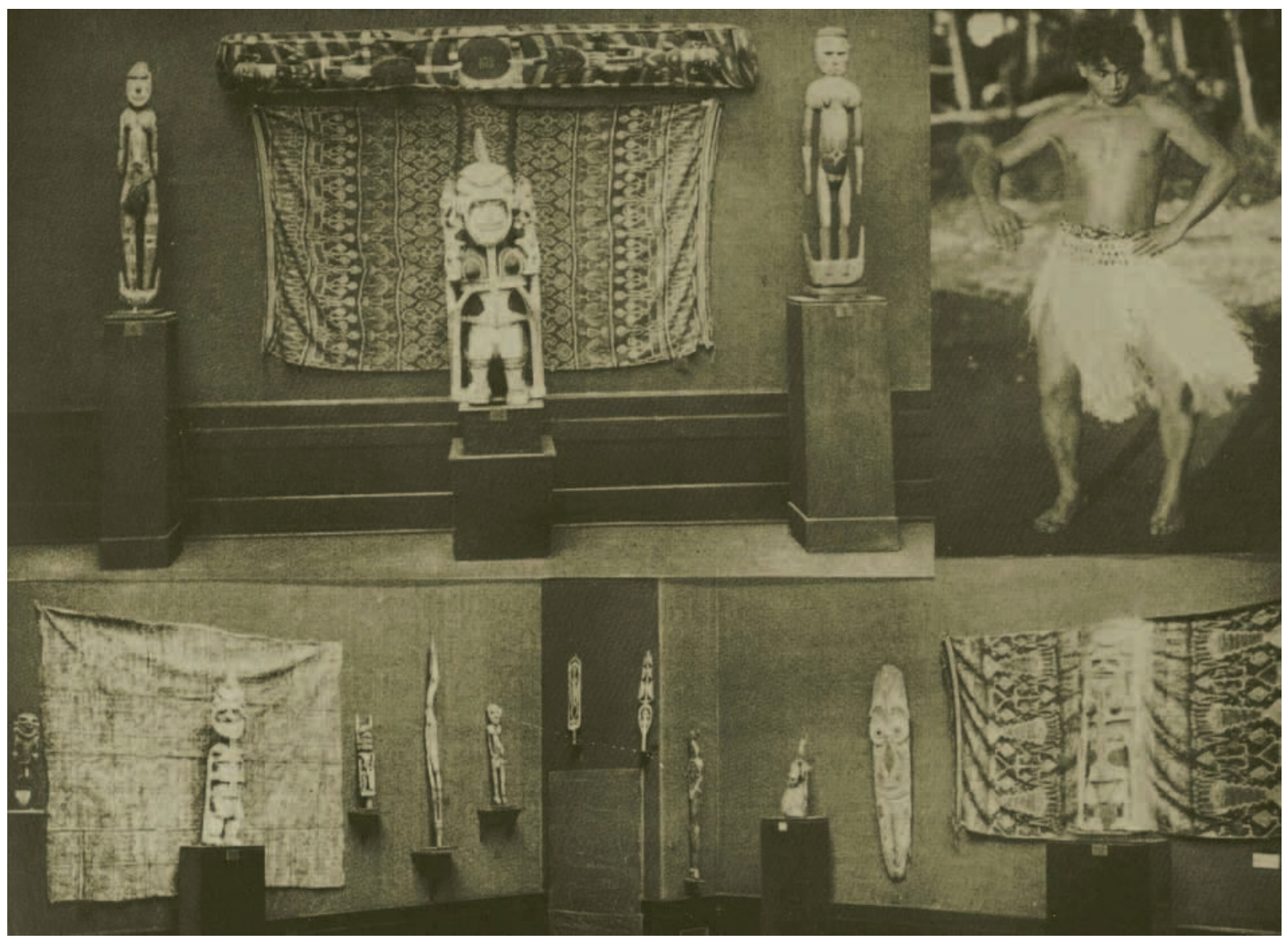

Fig. 5 Vue de l'exposition sur les statuaires des mers du Sud à Zurich, in Omnibus. Almanach auf das Jahr 1932. 